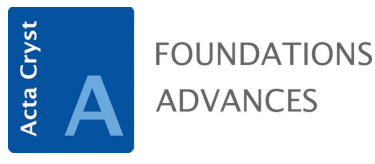

ISSN 2053-2733

Keywords: book review; X-ray crystallography.

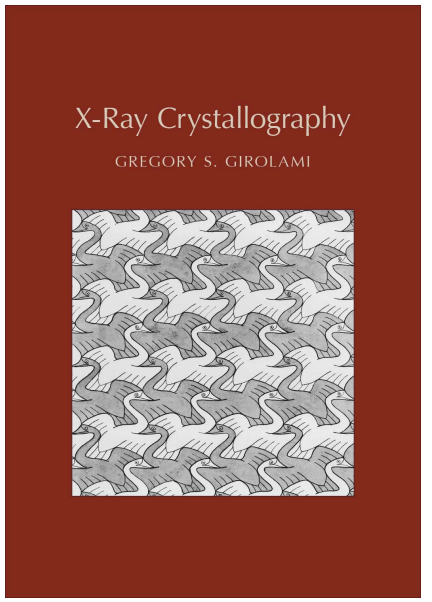

(C) 2016 International Union of Crystallography

\section{X-Ray Crystallography. By Gregory S. Girolami. University Science Books, 2015. Pp. 500. Price (hardcover) USD 88.00. ISBN 978-1-891389-77-1.}

\author{
Massimo Nespolo*
}

Université de Lorraine, CRM2, UMR 7036, Vandoeuvre-les-Nancy, F-54506, France, and CNRS, CRM2, UMR 7036, Vandoeuvre-les-Nancy, F-54506, France. *Correspondence e-mail: massimo.nespolo@crm2.uhp-nancy.fr

$X$-Ray Crystallography is a textbook (available also as an e-book) with a wide wingspan, composed of three sections covering basic symmetry notions, X-ray diffraction, and structure solution and refinement methods. It clearly addresses a public having essentially a background in chemistry and is written in a clear, didactic style. Because not everything can be treated even in a quite sizeable text (500 pp.), a clever choice has been made not to repeat what is already available ad libitum in countless books. The content concentrates instead on those subjects that too often are either absent or presented with a too specialistic approach for the intended audience. The result is an up-to-date textbook, pleasant and easy to read, which indeed responds to a clear need. Each chapter presents a topic with a good compromise between completeness and synthesis, supplemented by a series of exercises (with solutions available from the publisher). Unfortunately, a few but important slips, discussed below, cast some shadows on an otherwise excellent text. The choice of not including the metric tensor is worth criticism: a few long formulas that show up as a sort of deux ex machina would have been self-evident had the metric tensor been used.

The first section, Symmetry and space groups (139 pp.), is divided into 12 chapters and aims at giving a robust, although necessarily limited, background to point- and spacegroup theory with special emphasis on those aspects that a chemist needs to master in their daily work (the author had actually sent me a previous version of this section to get my critical opinion and many, although not all, of my remarks were apparently been considered useful enough to modify the text before it went to press). After a brief but appreciable historical introduction (Chapter 1), point symmetry and point groups are presented in two chapters, the rest being devoted to plane and space groups. One can particularly appreciate the distinction between symmetry element and geometric element that is ignored in so many texts but clearly explained here. It is therefore even more surprising and hard to understand the presence of some terminological liberties and imprecisions. The unnecessary neologism 'travel symmetry operation' has been introduced to indicate fixed-point-free operations (i.e. operations including an intrinsic translational component). The term 'mirror', used both for the symmetry element and the operation (instead of 'reflection'), is a misuse of language that could and should have been avoided. The difference between inversion point and centre of symmetry, incorrectly treated as synonyms, should have been explained. One may also wonder why orthographic, instead of the standard stereographic, projections are used as a graphic tool to explain point symmetry operations (p. 19ff); why the generators (which are operations) of a group have been called 'characteristic symmetry elements' (p. 29); why cyclic groups are not called as such (p. 30). Despite these defects, the presentation is pedagogical yet more rigorous than in many other textbooks.

Periodicity is the object of the fourth chapter, where the notions of lattice and unit cell are introduced. Chapter 5 describes travel (sic) symmetry operations where a significant slip occurs in footnote 5 on p. 60 , the $e$ glide being defined as two glide planes mutually perpendicular: the confusion between symmetry operation and symmetry element is unexpected and surprising. Chapters 6 and 7 introduce two-dimensional and threedimensional lattices, respectively. In the lower crystal families, the lack of restrictions imposed by symmetry is correctly indicated by leaving the corresponding cell parameter 'arbitrary', instead of assigning it a value 'different from', a typical mistake affecting many other textbooks. Chapter 8 introduces plane groups in a graphical and intuitive way, 
without the use of matrices: the same is true in the following chapters on space groups. Although drawings and analogy are powerful didactic tools, there is a clear limit to what can be obtained in this way; the complete absence of any matrix treatment is a clear shortcoming here.

Chapter 9 has the title 'Equivalent positions' and discusses general and special Wyckoff positions, but actually starts with a detailed description of chirality and handedness, concepts of paramount importance in chemistry. Unfortunately, this does not prevent a serious error in the following chapters, where confusion between the chiral nature of a structure and that of its space group occurs. Chiral groups are those forming the 11 enantiomorphic pairs (mentioned, but not named, on p. 135) and not the 65 types of space groups containing only operations of the first kind, which are instead known as Sohncke groups. [This problem was addressed in Nespolo (2015a), but apparently without much effect.]

The first section ends with three chapters on space groups, where the reader is guided through a step-by-step interpretation of the space-group diagrams with several general (abstract) and concrete (molecular) examples, but without any real calculation.

Section 2, X-rays and diffraction (152 pp.), consists of 15 chapters and overall is one of the most efficient presentations of the subject I have recently read, avoiding the typical traps of cloning old stuff. After a short introduction about the generation of X-rays (Chapter 13), where the essential matter is presented in a clear and concise way, the reader is taken on a short but effective journey through goniometers, (modern) detectors and data treatment, to land on the island of data reduction, where concrete directions for obtaining exploitable data from the measured intensities (and the physical reasons behind the necessary corrections) are presented. At this point one would have expected an explanation about Miller indices from the previous sections; but the text has not covered morphological aspects and thus Miller indices are still unknown, so the author is obliged to refer to Chapter 21 (we are still in Chapter 14). The poor expression 'lattice defects' (p. 173) to indicate what are actually structural defects echoes some die-hard laboratory jargon.

The next four chapters present diffraction of X-rays from an electron, an atom and arrays of atoms. Mathematical details are kept to a minimum, yet the presentation is clear and detailed. Resonant scattering is introduced right after Rayleigh scattering, making it a natural extension of a simplistic description, rather than an 'anomalous' phenomenon. Laue equations are introduced via the diffraction cones (à la Buerger), which leads naturally to the reciprocal space and lattice. The four reflection indices (read Laue indices) for trigonal and hexagonal crystals are confined to a footnote on p. 222, which is insufficient and unsatisfactory. A convincing presentation requires the use of Bravais-Miller indices but, as already noted, morphology is not covered in this textbook.

Chapter 21 introduces Bragg's law and $d$ spacing, and here Miller indices finally appear, with an unavoidable reference to the notions of lattice planes, crystal faces and interplanar distances. With only six and a half pages and despite the already manifest talent of the author for concentrating quite a lot of notions in a short space, here the reader is left gasping for breath. To be noted is the incorrect statement (p. 230) according to which Miller indices for crystal faces are always reduced to their lowest terms, as this is not true for lattice planes in centred cells (Nespolo, 2015b); the same actually applies to a crystal face, which is the last plane of the family. Also to be noted is the use of parentheses for reflection (Laue) indices: this use should actually be restricted to Miller indices, to indicate families of lattice planes.

Chapter 22 deals with the limiting sphere (the Ewald sphere is instead relegated to Appendix C), resolution and indexing. The use of the metric tensor would have clarified the origin of the long and mysterious formula of $1 / d$. Apart from these questionable choices, the chapter is clear, concise and easy to follow. One may remark that resolution is defined in angstroms only, whereas the complementary definition $\AA^{-1}$, directly related to the region of the reciprocal space explored and to the decay of the atomic scattering factor, is commonly used by the experimentalist: in a practical textbook one may expect that the reader's attention is drawn to these details. The next chapter on structure factors follows naturally and is complemented by an introduction to Argand diagrams, which will be used later. It prepares the way for a chapter on Laue classes and symmetry-related data, with the emphasis on the meaning of data merging.

Chapter 25, 'Centrosymmetry and chirality', is of paramount importance for chemists in the audience, but is unfortunately handicapped by the confusion between chirality of structures and chirality of groups. For example, the statement (p. 266) that among the ' 65 polar space groups' there are both 'chiral' groups, like $P 1$, and 'achiral' groups, like $P m$, does not make sense. Firstly, polar groups (which contain at least one polar direction) are 68 in number, not 65 (the latter are the Sohncke groups, here incorrectly called 'chiral'). Secondly, neither $P 1$ nor $P m$ are chiral groups (they do not belong to the 11 pairs of enantiomorphic groups). If the reader survives this terminological storm, they will then land on a happy island where a smiling team of absolute configurations and absolute structures greet them. It's a pity that when the wrong absolute structure is picked up by the structure-solution software and the user needs to invert it, the solution in some cases is just described as 'more complicated'. Here (but later too) the use of normalizers would have brought the necessary light, but unfortunately this fundamental topic even today is considered too specialist.

Chapter 26 introduces reflection conditions with a slip at the very beginning, where translations centring non-primitive unit cells are considered 'operations [that] involve a translation component that is not a lattice vector for the chosen unit cell'. Quite obviously, a centring vector definitely is a lattice vector, although not a basis vector. The intuitive description of integral reflection conditions will certainly be rather obscure for a newcomer in the field; more convincing is the following description as a result of destructive interference. The analytical derivation via the structure factor is classical stuff, but when it comes to zonal and serial conditions the description is 
affected by incorrect use of the Miller indices (Fig. 26.4), where planes of the same family with both coprime and noncoprime indices - like (001) and (002) - coexist as if they belonged to different families of lattice planes. The section ends with a chapter about space-group determination from the extinction symbol and intensity distribution.

The last section, Solving and Refining Crystal Structures (157 pp.), includes 15 chapters with a very practical approach, spanning from Fourier theory, to solution methods (trial-anderror, charge flipping, Patterson, heavy atom, protein structures, direct methods, modelling electron density), to refinement strategies and twinning, and ending with chapters on powder X-ray diffraction, electron and neutron diffraction. Almost $100 \mathrm{pp}$. are devoted to methods, and two entire chapters to direct methods: apart from specialistic texts, this fundamental topic often receives insufficient space, whereas here we get a clear picture, although without all the details that may appear somewhat intimidating for the target audience. A few words about multipolar refinement and the maximum-entropy method would have had their place here. The reader will especially enjoy Chapter 40 ('mistakes and pitfalls'), where several cases of wrong structure reports from the literature are discussed. I cannot refrain from pointing out a mistake on p. 428 , where the lattice built on a supercell is called a superlattice instead of sublattice: being based on a larger cell, the lattice has lost translations and the group of translations is therefore a subgroup of that of the original lattice; it cannot evidently be a superlattice! Another curious mistake occurs in the first of the two chapters (No. 34) devoted to direct methods where a one-dimensional example is chosen (p. 361) but treated with three Miller indices (although two are zeroed): if the space has one dimension, then obviously also its reciprocal is one-dimensional. The description of twinning here and there is imprecise, with confusion between twin law and twin operation and the use of non-crystallographic rotations, arising from the misorientation of the reciprocal lattices (the misorientation is kept into account in the twin obliquity or misfit, but the twin operation is crystallographic) (Nespolo, 2015c). A clear misunderstanding occurs in the example of the twin of ornithine- 5 '-monophosphate dehydrogenase, where a 'non-standard space group $C 2_{1}$ ' is mentioned. In a $b$-unique monoclinic setting, as in this case, $C 2_{1}$ is the same as $C 2$ : a $C$-centring vector added to $P 2_{1}$ generates twofold axes. No mention of this is made in the original article (Wittmann \& Rudolph, 2007), where twinning of a $P 2_{1}$ crystal simulating $C 222_{1}$ symmetry is presented. Apart from these errors, the section is very well written and the special emphasis put on methods typically used in protein crystallography (SIR, MIR, SIRAS, MAD etc.) is a valuable aspect not often present in textbooks targeting a readership of chemists. One can, however, once again regret the absence of normalizers, which would have been of much help in the example on p. 338 as well as in the discussion about trial-and-error methods.

A set of six short appendices (vector and complex-number tutorials, the Ewald sphere, the analytical derivation of atomic scattering factors and of Patterson function, and an in-class demonstration of diffraction), followed by a (much too short) bibliography and a detailed index end the book.

Some typographical errors occur here and there in the text. An erratum is available from the publisher (http://www. uscibooks.com) which corrects most of these. Besides those, one should remark on the dots in the graphical symbols of glide planes (Fig. 5.5), which are far too big; some confusion between periodicity and dimension occurring in Chapter 6, where three-dimensional diperiodic structures are described as two-dimensional (p. 67); and the presence of only two equivalent directions, instead of three, for the second and third positions in the trigonal and hexagonal crystal system.

Overall, and despite some defects pointed out above, this is perhaps one of the best textbooks addressing a readership of chemists published in recent years. If an updated version is planned, one can only encourage the author to correct the errors described above, and add some matrix algebra and a chapter on normalizers: the result would be a reference text for several years to come.

\section{References}

Nespolo, M. (2015a). Cryst. Res. Technol. 50, 413.

Nespolo, M. (2015b). J. Appl. Cryst. 48, 1290-1298.

Nespolo, M. (2015c). Cryst. Res. Technol. 50, 362-371.

Wittmann, J. G. \& Rudolph, M. G. (2007). Acta Cryst. D63, 744-749. 